\title{
Prospective study of hypothalamo-hypophyseal dysfunction in children and adolescents following traumatic brain injury
}

\author{
David Krahulik, , Darina Aleksijevic ${ }^{b}$, Vratislav Smolka ${ }^{b}$, Eva Klaskova ${ }^{b}$, Petra Venhacovab ${ }^{b}$, Miroslav Vaverka ${ }^{a}$, \\ Vladimir Mihal ${ }^{b}$, Jirina Zapletalova ${ }^{b}$
}

\begin{abstract}
Background and Aims. Retrospective studies of TBI have found a neuroendocrine dysfunction following traumatic brain injury in 23 to $60 \%$ of adults and 15 to $21 \%$ of children. Our aims were to determine the prevalence of hypothalamo-hypophyseal dysfunction in children following brain injury, assess its relationship to the type of injury and the course of the acute post-traumatic phase.

Patients and Methods. Body development (growth, pubertal development, and skeletal maturity) were evaluated in 58 patients ( 21 girls) after a brain injury rated 3 to 12 on the Glasgow Coma Scale (GCS). The patients underwent standard endocrine tests - TSH, fT4, IGF-1, PRL, morning cortisol, FSH, LH, and testosterone in boys and estradiol in girls - in the early post-traumatic period ( 2 to 14 days; T0) and at 3, 6, and 12 months after the injury (T3, T6, and T12). Dynamic tests were carried out in patients with abnormalities in their clinical examination and/or laboratory results. An MRI was performed on all patients at T12.

Results. The median age at the time of injury was 11.3 ( 0.5 to 18.7 ) years. Of the 58 patients, 23 had GCS $<8$, corresponding to severe brain injury. At T0, diabetes insipidus (DI) was diagnosed in 12 patients, and the syndrome of inappropriate antidiuretic hormone secretion (SIADH) was found in 4 patients. Frequent hormonal changes simulated central hypothyroidism (in $45 \%$ of patients) and hypogonadotropic hypogonadism (in $25 \%$ of adolescents who were already pubertal at the time of injury $>$ Tanner II). Examination at T3 $(n=58)$ confirmed a combined pituitary hormone deficiency in two boys and DI in another one. At T6 $(n=49)$, hormonal dysfunctions were diagnosed in two boys (precocious puberty and growth hormone deficiency). At T12 $(n=39)$, a new endocrine dysfunction was diagnosed in five patients (growth hormone deficiency in two, hypogonadotropic hypogonadism in two, and in one patient, already diagnosed with a growth hormone deficiency, central hypothyroidism, as well). Brain MRI revealed an empty sella in two patients with growth hormone deficiency. Patients with GCS $<8$ had more symptoms of SIADH or DI in the early post-traumatic period $11 / 23$ vs. patients with GCS of 8 to $13(4 / 35)$, and more frequent hormonal disorder $(6 / 23)$ than individuals with moderate trauma (3/35), $P=0.0135$. The incidence of endocrine dysfunction at T0 significantly correlated with the severity of injury $(P=0.05)$, but it was not an indicator for the development of a late hormonal disorder. Conclusion. Within a year after injury, a hormonal disorder was found in $17.6 \%$ of the patients. Neuroendocrine dysfunction as a late consequence of craniocerebral trauma in children and adolescents was less frequent than in adults. Risk factors for its development are the gravity of the injury, brain scan pathology, and possibly the development of DI, SIADH, or CSWS in the acute post-traumatic phase.
\end{abstract}

Key words: traumatic brain injury, children, post-traumatic hormonal disorder, growth hormone deficiency, precocious puberty, risk factors

Received: June 17, 2016; Accepted with revision: September 7, 2016; Available online: September 19, 2016 http://dx.doi.org/10.5507/bp.2016.047

${ }^{a}$ Department of Neurosurgery, University Hospital Olomouc and Faculty of Medicine and Dentistry, Palacky University Olomouc, Czech Republic

${ }^{b}$ Department of Pediatrics, University Hospital Olomouc and Faculty of Medicine and Dentistry, Palacky University Olomouc, Czech Republic Corresponding author:David Krahulik, e-mail:david.krahulik@fnol.cz

\section{INTRODUCTION}

Cerebral injuries in children and adolescents, with an average incidence of 250:100,000 a year, are one of the most common causes of mortality and severe morbidity. The frequency is twice as high in boys than in girls. The largest group is composed of adolescents 15 to 19 years of age, and the second largest group is composed of children under 5 years of age. The causes are mostly tumbling and physical abuse among younger children ("shaken baby syndrome" leads to the most serious injuries in more than
$50 \%$ of children between 0 and 2 years), sports activities among older children, and traffic accidents among adolescents $^{1,2}$. The consequences of medium to severe cerebral injury are often very serious, from physical handicaps to psychological problems with obvious social implications.

Hormonal dysfunctions due to brain injury were long considered a rarity ${ }^{3,4}$, although histopathological studies performed post mortem in the 1970s and 1980s proved the hypothalamus and hypophyseal regions to be fairly vulnerable to exterior sources of harm ${ }^{5-7}$. 
The disruption of the integrity and functioning of hypothalamic-pituitary structures could lead to temporary, as well as permanent, neuroendocrine dysfunction and influence patient morbidity.

Temporary failure of water management - diabetes insipidus (DI) - is a frequent and anticipated complication of transitory dysfunction of the neurohypophysis, as is the syndrome of inappropriate antidiuretic hormone (SIADH) . Transient hormonal changes, comparable to changes of the hypothalamo-hypophyseal axis, have also been observed in other forms of critical conditions ${ }^{3,8}$.

Permanent endocrine dysfunction manifesting months or years after the injury was once considered rare. This opinion has changed over the last decade, as numerous studies conducted on adults established the prevalence of post-traumatic hypopituitarism in 23 to $69 \%$ of the cases (Table 1) (ref.9-15). Much less is known about neuroendocrine dysfunctions in the pediatric population than about the adult population. The results of retrospective studies indicate a lower frequency of hormonal deficiency in children than in adults (Table 2) (ref. ${ }^{16-21}$ ).

We present the results of a prospective study of hormonal functions in children and adolescents hospitalized in the ICU of the Department of Pediatrics of the University Hospital in Olomouc after a medium to severe brain injury. The study was approved by the ethics committee of the University Hospital Olomouc and we obtained the written consent of the legal representatives of all of the patients.

\section{PATIENTS AND METHODS}

\section{Patients}

The prospective group included 58 children and adolescents ( 21 girls) who were hospitalized between January

Table 1. Post-traumatic hypopituitarism in adults.

\begin{tabular}{lccccc}
\hline Author & $\begin{array}{c}\text { Number of } \\
\text { patients }\end{array}$ & $\begin{array}{c}\text { Age } \\
\text { (years) }\end{array}$ & $\begin{array}{c}\text { Time from the injury } \\
\text { (months) }\end{array}$ & $\begin{array}{c}\text { Prevalence of hormonal } \\
\text { dysfunction (\%) }\end{array}$ & Ref. \\
\hline Kelly & 22 & $20-52$ & $3-254$ & 36.4 & 9 \\
Liberman & 70 & $18-58$ & $1-254$ & 68.5 & 10 \\
Bondanelli & 50 & $20-87$ & $12-60$ & 54.0 & 11 \\
Aimaretti & 100 & $37 \pm 2$ & 3 & 35.0 & 12 \\
Agha & 102 & $15-65$ & $6-36$ & 28.4 & 13 \\
Klose* $_{\text {Krahulík* }}$ & 46 & $19-65$ & $3-12$ & 10.8 & 14 \\
\hline
\end{tabular}

* prospective study

Table 2. Post-traumatic hypopituitarism in children.

\begin{tabular}{|c|c|c|c|c|c|c|}
\hline Author & $\begin{array}{l}\text { Number } \\
\text { of patients } \\
(\mathrm{M} / \mathrm{F})\end{array}$ & $\begin{array}{l}\text { Age * } \\
\text { (years) }\end{array}$ & $\begin{array}{l}\text { Time from } \\
\text { the injury } \\
\text { (months) }\end{array}$ & Hormonal dysfunction & $\%$ & Ref. \\
\hline Sockalosky & $33(22 / 11)$ & $\begin{aligned} \mathrm{F} & <8.5 \\
\mathrm{M} & <10.0\end{aligned}$ & $\begin{array}{l}3-76 \\
9-18\end{array}$ & 7 precocious puberty & $21 \%$ & 16 \\
\hline Goldman & $21(11 / 10)$ & $3.0-18.5$ & $4-132$ & $\begin{array}{l}1 \text { precocious puberty } \\
3 \text { osteal age acceleration }\end{array}$ & $19 \%$ & 17 \\
\hline Niederland & $26(17 / 9)$ & $1.5 \pm 0.8$ & $30.6 \pm 8.3$ & $\begin{array}{l}42 \% \text { STH deficiency } \\
12 \% \text { TSH deficiency } \\
\text { (34\% lower hydrocortisol) }\end{array}$ & $61 \%$ & 18 \\
\hline Einaudi & $22(19 / 3)$ & $1.2-18.0$ & $7-87$ & $\begin{array}{l}1 \text { precocious puberty } \\
1 \mathrm{GH}, \text { ACTH, TSH deficiency } \\
1 \mathrm{FSH}, \mathrm{LH} \text { deficiency } \\
1 \mathrm{GH} \text { deficiency }\end{array}$ & $18 \%$ & 19 \\
\hline Poomthavorn* & $54(31 / 23)$ & $1.0-14.8$ & $1-115$ & $\begin{array}{l}3 \mathrm{GH}, \mathrm{ACTH}, \mathrm{TSH} \text { deficiency } \\
1 \mathrm{GH} \text { deficiency } \\
1 \text { precocious puberty } \\
1 \text { TSH deficiency } \\
3 \text { lower hydrocortison }\end{array}$ & $16.7 \%$ & 20 \\
\hline Aleksijevič & $30(18 / 12)$ & $0.4-14.7$ & $16-33$ & $\begin{array}{l}1 \text { deficit GH and TSH } \\
1 \text { deficit GH } \\
1 \text { deficit FSH and LH } \\
2 \text { precocious puberty }\end{array}$ & $16.7 \%$ & 21 \\
\hline
\end{tabular}


1, 2009, and September 1, 2012 after a medium to severe brain injury rated 3 to 12 on the Glasgow Coma Scale (GCS).

Patients with a history of severe chronic illnesses and patients receiving long-term hormonal treatment (including birth control) were excluded from the study. The causes of injury were a traffic accident in $50 \%$, a tumble in $35 \%$ (half during sport activities), and some other cause in $15 \%$ (kick from a horse, from a ram, hanging oneself, abuse). The median age of the patients was $11.3(0.5-18.7)$ years; 23 (38\%) were in puberty (>Tanner II) at the time of injury. The severity of the injury was evaluated according to GCS after a basal resuscitation: 35 patients had a medium injury (GCS 8 to 12 ) and 23 patients a severe injury $(\mathrm{GCS}<8)$.

At the time of the injury, all of the patients underwent a brain examination with computerized tomography (CT) or magnetic resonance imaging (MRI). Severe pathological findings, including cerebral edema, subdural hematoma, subarachnoid hemorrhage, epidural hemorrhage, diffuse axonal injury, midline structures shift, and cranial base fracture, were diagnosed in 21 (37\%) of the patients. The clinical condition of 14 of these patients required artificial respiration for 8 (1-150) days on average.

\section{Clinical examination}

A physical examination, including the following data: current height, weight, body mass index (BMI), growth velocity, and degree of pubertal development according to the Tanner scales ${ }^{22,23}$ was performed in the early post-traumatic period (2-14 days, T0) and at 3, 6, and 12 months after the injury (T3, T6, and T12). The body growth and BMI prior to the injury were evaluated based on data retrieved from individual documentation compiled by the general physicians of the individual patients. The results were compared with the standards for the Czech population released in 2001 and set as a standard deviation score (SDS) for age and gender ${ }^{24}$. At T0 and T12, biological maturity was evaluated according to bone maturity from a left arm and wrist X-ray following Greulich and Pyle ${ }^{25}$. The observations included a 24 -h fluids balance.

\section{Laboratory evaluation}

Basic biochemical and hormonal screening (after fasting from 7 p.m. to 9 a.m.) was performed at T0, T3, T6, and $\mathrm{T} 12$, and included the levels of mineralogram, glycemia, osmolality of serum and urine, thyroid stimulating hormone (TSH), free thyroxine (fT4), free tri-iodothyronine (fT3), morning cortisol, adrenocorticotropic hormone (ACTH), insulin-like growth factor 1 (IGF-1), prolactin (PRL), follicle-stimulating hormone (FSH), luteinizing hormone (LH), and estradiol in girls and testosterone in boys.

In cases of pathological results or growth disturbance (including bone age acceleration or retardation more than \pm 6 months) or inappropriate sexual maturation to age, standard stimulating tests were performed, verifying the function of specific hormonal axes ${ }^{25}$.

In cases of low levels of IGF-1 (-1.5 SDS), we verified the secretion creation of growth hormone $(\mathrm{GH})$ by a stimulating test with pyridostigmine and L-dopa, as well as an insulin-induced hypoglycemia test (ITT). An ITT was conducted only in patients with normal neurological findings. In boys older than 9 years and in girls older than 8 years, we used estrogen priming.

The level of gonadotropins ( $\mathrm{FSH}$ and $\mathrm{LH}$ ) was correlated to the age, and in adolescent girls to the menstrual cycle phase. In cases of doubt, its secretion was evaluated with the aid of gonadoliberin stimulation (LHRH) and the results were related to the patient age and sex.

A low level of TSH and a concurrent low level of fT4 indicated central hypothyreosis. A low level of ACTH and morning hydrocortisone, as well as a low response of hydrocortisone in ITT and after ACTH application, indicated central hypocorticism. At T12, a brain MRI was performed on all patients if it had not been required earlier by their condition.

\section{Statistic evaluation}

The data are presented as median (range). Comparison between groups was performed using the Fischer test. To evaluate the possible predictive factors in pituitary insufficiency, a regression analysis method was used. For statistical purposes, $P<0.05$ was considered a significant level. We used GraphPad Prism software, version 1.5.

\section{RESULTS}

In the acute phase (T0), 58 children and adolescents were examined. In $45 \%$ of the patients, the laboratory changes indicated central hypothyreosis; in $25 \%$ of adolescents, the changes indicated hypogonadotropic hypogonadism. The level of PRL was increased in $35 \%$ of the patients, and in $10 \%$ there was an increase in the cortisol level and ACTH. The level of IGF-1 decreased in 5 patients. DI was diagnosed in 12 patients (with a transition to SIADH in one of them) and SIADH was diagnosed in four of the patients. In severe cases, acute electrolyte disturbances may even be life threatening, and they must be followed carefully. A higher frequency of hormonal changes was observed in the patients with a severe injury $(20 / 23)$ than in those with a moderate injury $(11 / 35) \quad(P<0.05)$. However, these changes were transitory in all patients, and at the time of release to home care their hormonal status was physiological. The exception was two very young boys diagnosed with severe trauma in whom a combined deficiency of pituitary hormones ( $\mathrm{GH}$, TSH, and ACTH) was proven and prevailed up to their respective deaths in the third and fifth month after trauma. They were diagnosed with apallic syndrome, and were administered L-thyroxine and hydrocortisone. Individual findings on the CT or MRI scans did not indicate a specific endocrine disorder.

At 3 months (T3) after the injury, the results of 56 of the 58 patients showed normal auxologic and hormonal findings. The two remaining patients had apallic syndrome and combined pituitary hormone deficiency. They were the only two patients in whom endocrinopathy persisted even after the end of the acute phase. 
Another patient, who in the third month showed signs of endocrinopathy, was a boy who became constantly thirsty and was significantly wetting the bed after the injury, but his hormone levels at the time of discharge were normal; these subjective problems started only shortly after his discharge to home care. A concentration test with ADH revealed central DI, and so the substitution of ADH was initiated. The patient had transient DI during the acute phase, too, but it later transformed into transient SIADH. His enuresis was initially attributed to posttraumatic stress dysfunction. However, in roughly $6 \%$ of patients, DI with consequent SIADH transforms back into a permanent DI (a "triple reaction").

At 6 months (T6) after the injury, 49 children were examined, and 6 patients underwent a stimulating test based on pathological laboratory examinations verifying the $\mathrm{GH}$ secretion. A GH deficiency was confirmed in only one patient, for whom a different GH treatment was started. With three children, there was a suspicion of precocious puberty, but the diagnosis was verified after LHRH in only one patient. None of the patients showed any symptoms of clinical hormonal dysfunction.

An examination at 12 months (T12) after the injury was performed on 39 patients $(67.5 \%$ of the original group). From previous visits, hormonal dysfunction was confirmed in three patients. Stimulating tests were indicated for a further 12 children. The reasons were the growth and retardation of bone age, menstrual cycle disorder, or pathology laboratory results. Two cases of moderate GH deficiency were confirmed, and in two girls partial hypogonadotropic hypogonadism (both were experiencing irregular menstrual cycles with oligomenorrhea). One boy who was treated with GH 6 months after the trauma because of GH deficiency also had manifestations of central hypothyreosis (low fT4 and TSH).

A pathological finding in the hypophyseal region (empty sella) was confirmed in two patients after MRI examination. Both were diagnosed with hormonal dysfunction prior to the MRI examination.

\section{Early and late post-traumatic period comparison}

Changes resembling imitated pituitary hormone dysfunction were observed in $65 \%$ of patients in the early phase after brain trauma. In $45 \%$, central hypothyreosis was observed, and in $25 \%$ of adolescents, hypogonadotropic hypogonadism. The level of PRL increased in 35\% of patients, and in $10 \%$ there was an increased level of cortisol and ACTH. The level of IGF-1 decreased in five patients. In nearly all patients, these changes were of a transitory character (except for two with apallic syndrome).

In nine patients, late manifestation of hormonal dysfunction occurred at T3 to T12. GH deficiency was confirmed in three patients (in one case combined with central hypothyreosis). One boy experienced precocious maturation, and two girls had hypogonadotropic hypogonadism (Table 3). DI and SIADH were observed in 16 patients. In one of them, DI converted to the permanent type.

The prevalence of hormonal disorders in our study was $17.9 \%$ (after excluding the two deceased patients, it was $11.6 \%$ ). The loss of patients for observation between T6 and T12 is probably because if no hormonal disorder was detected within 6 months after the injury and the child had no clinical symptoms, the parents did not bring the child for further follow-up visits. This is consistent with observations in other prospective studies.

\section{Risk factors influencing late hormonal dysfunction}

Patients with hormonal dysfunction were younger than the average age of the group, i.e. $9.1(0.5-17.1)$ years. There was no difference in the development of hormonal dysfunction between boys and girls (6/37 : 3/21). Patients were more likely to have hormonal dysfunction after a severe injury ( 6 out of 23 ) than after a medium injury (3 out of 35) $(P=0.135)$.

The majority of patients with hormonal dysfunction had pathological findings in brain imaging following the

Table 3. Patients with confirmed hormonal dysfunction.

\begin{tabular}{|c|c|c|c|c|c|c|}
\hline $\begin{array}{l}\text { Patient/ } \\
\text { gender }\end{array}$ & $\begin{array}{l}\text { Age at time of } \\
\text { injury (years) }\end{array}$ & GCS & $\mathrm{CT} / \mathrm{MRI}$ & Acute phase (T0) & $\begin{array}{l}\text { Late phase hormonal } \\
\text { dysfunction (time) }\end{array}$ & MRI (T12) \\
\hline $1 / \mathrm{M}^{*}$ & 0.5 & 3 & BS, SDH & $\begin{array}{l}\downarrow \mathrm{TSH}, \mathrm{ACTH}, \\
\text { STH, SIADH }\end{array}$ & $\downarrow$ TSH, ACTH, STH (T3) & - \\
\hline $2 / \mathbf{M}^{*}$ & 14.9 & 3 & BS, SDH, DAH & $\begin{array}{l}\downarrow \mathrm{TSH}, \mathrm{ACTH}, \\
\text { STH, } \uparrow \text { PRL } \\
\text { SIADH }\end{array}$ & $\downarrow$ TSH, ACTH, STH (T3) & - \\
\hline $3 / \mathrm{M}$ & 7.9 & 8 & Base fracture, BS & DI/SIADH & DI (T3) & Norm. \\
\hline $4 / \mathrm{M}$ & 6.8 & 8 & SAH & - & $\downarrow$ IGF 1, STH (T6), TSH (T12) & Empty sella \\
\hline $5 / \mathrm{M}$ & 8.1 & 10 & Normal & - & $\uparrow \mathrm{FSH}, \mathrm{LH}$, testosterone (T6) & Norm \\
\hline $6 / \mathrm{F}$ & 6.1 & 7 & SAH & DI & $\downarrow$ IGF 1, STH (T12) & Norm. \\
\hline $7 / \mathrm{M}$ & 3.7 & 6 & Normal & $\uparrow \mathrm{PRL}$ & $\downarrow$ IGF 1, STH (T12), & Empty sella \\
\hline $8 / \mathrm{F}$ & 17.1 & 10 & Normal & - & $\downarrow$ FSH, LH, E2 (T12) & Norm. \\
\hline $9 / \mathrm{F}$ & 15.6 & 12 & SAH & - & $\downarrow$ FSH,LH,E2 (T12) & Norm. \\
\hline
\end{tabular}

* patients with apalic syndrome

BS - brain swelling, SDH - subdural hematoma, SAH - subarachnoideal hemorrhage, DAI - diffuse axonal injury 
injury (6 out of 9 patients). Symptoms of water and electrolyte disturbance in the early post-traumatic phase were more common in patients with hormonal dysfunction ( 4 out of 9 patients), compared to 12 out of 49 patients $(P<0.05)$ without hormonal dysfunction

Table 4 shows the comparison of patients with and without confirmed hormonal deficiency.

\section{DISCUSSION}

Brain injuries in children represent a significant medical problem worldwide. Although the numbers of surviving children are higher than those of adults, the post-traumatic complications can be more severe, as the injury affects an organism with unfinished body development. Post-traumatic hypopituitarism (PTH) has long been described only in case reports. Numerous recent studies have evaluated (mostly retrospectively) the incidence of PTH in adult patients and in children ${ }^{2,12-15,26}$. The prevalence of a hormonal dysfunction is, according to the results, highly variable: between 9 and $63 \%$ of the adult population and between 10 and $61 \%$ in children. The interval of the manifestation of a hormonal deficiency also varies (Tables 3 and 4).

There has been increasing evidence that while hormonal changes in the acute post-traumatic phase are mostly transitory and do not require specific long-term treatment, late unidentified hypopituitarism can significantly influence the recovery phase as well as patient morbidity. The premise for the accurate diagnosis of endocrine dysfunction is the adaptation of hormonal levels to the age of patients and their eventual medication (for example hormonal contraception). In adults, it is necessary to take into account the natural decrease in $\mathrm{GH}$ and sex steroid secretion during the patient's lifetime. The results of tests verifying $\mathrm{GH}$ secretion could be influenced in patients with other untreated hormonal dysfunctions, such as hypothyreosis or hypogonadism. A basal hormonal level formulation can be used in diagnosing thyrotropic, gonadotrophic, and perhaps corticotrophic insufficiency as well. To confirm STH deficiency, particularly in adults, simulating tests are required because in rare cases the level of IGF-1 could even fluctuate in physiological ranges.

Table 4. Comparison of patients with and without hormonal dysfunction.

\begin{tabular}{lcc}
\hline & $\begin{array}{c}\text { Patients } \\
\text { w PTH } \\
(\mathrm{n}=7)\end{array}$ & $\begin{array}{c}\text { Patients } \\
\text { w/o PTH } \\
(\mathrm{n}=32)\end{array}$ \\
\hline Average age & $9.1(0.5-17.1)$ & $11.9(1.9-18.7)^{*}$ \\
GCS < 8 & 4 & $13^{*}$ \\
CT/MRI finding in T0 & 4 & $11^{*}$ \\
DI/SIADH in T0 & 2 & 5 \\
CT/MRI finding in T12 & 2 & 0 \\
\hline
\end{tabular}

$* P<0.05$
Significant indicators of GH deficiency in child patients are growth rate deceleration and bone maturation delay.

It has been proven that the somatotropic and gonadotrophic axes, which are closely related to body growth and maturation, are affected in most cases. Thus, it should not be a problem to discover a growth dysfunction or an onset or duration of puberty. Nevertheless, evaluating the physiological development of children after severe trauma is often underestimated, and most care is primarily focused on motor-related or psychological disruptions. Difficult or impossible verticalization of a child sometimes hinders accurate height measurements. Delays in pubertal development or menstrual cycle disturbances are often put in relation to psychological post-traumatic dysfunctions. A brain injury can also, in contrast, induce a precocious onset of puberty, as in children with brain tumors or inborn development abnormalities of CNS, or after shunt surgery. Sockalosky and Goldmann described the occurrence of precocious puberty as a consequence of craniocerebral injury in their first studies concerning PTH in children. Even a significant hormonal dysfunction can be overlooked if it is hidden in neurological symptomatology, in difficult cooperation with the child during recovery, in a school results setback, or in behavioral disorders.

As in adult patients ${ }^{14}$, the results of our study confirmed a high incidence of transitory hormonal dysfunction in the early stage of a post-traumatic period. These changes ("sick syndrome" or "wasting syndrome") are often observed in other critical conditions, as well, and are merely an expression of adaptive changes of an organism rather than a consequence of structural damage. An acknowledged hypothesis is that the inhibition of the secretion of anabolic hormones - TSH, GnH, and GH leads to reduced energy consumption and the retention of metabolic resources which are necessary for the sustenance of primary organs, such as the brain, cardiovascular system, kidneys, etc. The levels of stress hormones such as PRL and ACTH tend to be increased ${ }^{1}$.

The results of this prospective study are described in Table 3. In three patients, the hormonal deficiency revealed in the early post-traumatic period persisted (two patients had apallic syndrome and died three and five months after the injury respectively).

In two children, we diagnosed hormonal changes six months after the injury and in another one 4 to 12 months after the injury.

The results are comparable with the findings of other studies and indicate the prevalence of hormonal dysfunction in about $20 \%$ of patients (Table 2 ). The exception is a retrospective study by Niederland et al, who expressed a suspicion of partial hormonal dysfunction in $61 \%$ of children, without any of the patients having corresponding clinical symptomatology. Even though the study proved a decreased secretion of $\mathrm{GH}$ in $42 \%$ of patients, the stimulating tests were not carried out with regard to the age of patients after typical estrogen priming. For this reason, the post-stimulative levels could have been falsely lower ${ }^{18}$.

The prevalence of hormonal dysfunction in our group of patients was $15.5 \%$; when we exclude the two deceased 
patients, it was $12 \%$. It is lower than in the groups of patients observed retrospectively later after the injury. It is likely for another hormonal dysfunction to occur later than the 12 months after the injury which is generally considered as a risk period. Factors likely to influence the development of a hormonal dysfunction include:

1. Severe brain injury (GCS 3 to 8 ).

2. Pathological finding during brain imaging at the time of injury.

3. Pathological finding on imaging hypothalomus and pituitary gland for 12 to 24 months after traumatic brain injury (pituitary hypoplasia, empty sella).

4. Younger age at the time of the accident.

5. Water and mineral disorder during the early post-traumatic phase (DI, SIADH, CSWS).

\section{CONCLUSION}

Long-term consequences after brain injuries are more complex than previously presumed. It is necessary to pay attention to the patients from the neurological, neurosurgical, rehabilitative, and psychological-psychiatric point of view, but also from an endocrinological one. Hormonal dysfunction as a consequence of brain injury is frequently marginalized, despite its high prevalence. In every child and adolescent patient after a craniocerebral injury, the relevance of their physical development in relation to their age should be evaluated, and at least a fundamental hormonal examination should be carried out, in the acute phase and over the long term, preferably 6-12 months, regardless of their clinical condition.

A group of patients who had experienced severe trauma, with a pathological finding after a brain screening at the time of injury and with DI, SIADH, or CWS in the early post-traumatic phase appear to account for a higher incidence of hormonal dysfunction. Adequate and timely hormonal treatment of diagnosed patients could significantly influence the recovery process and enhance their quality of life.

Author contributions: DK, DA: manuscript writing, data analysis, literature search; VS, EK, PV: taking care of patients at ICU unit; VM, JZ,MV: corrections of manuscript, final approval.

\section{REFERENCES}

1. Masson F, Salmi LR, Maurette P. Characteristics of head trauma in children: epidemiology and 5-years follow up. Arch Pediatr 1996;3:651-60.

2. Schneler AJ, Schields BJ, Hostetler SG. Incidence of pediatric brain injury and associated hospital resource utilization in the United States. Pediatrics 2006;118:483-92.
3. Cyran E. Hypophysisenschadigung durch Schadelbasis fracture. Dtsch Med Wochenschr 1918;44:1261-70.

4. Escamilla RF, Lisser H. Simmonds disease. J Clin Endocrinol Metab 1942;2:65-96

5. Ceballos R. Pituitary changes in head trauma. Analysis of 102 consecutive cases of head injury. Alabama J of Med Sciences 1996;3:185-98.

6. Crompton MR. Hypothalamic lesions following closed head injury. Brain 1971:94:165-72.

7. Harper CG, Doyle D, Hume AJ, Graham DI. Analysis of abnormalities in pituitary gland in nonmissile head injury: study of 100 consecutive cases. J of Clin Pathol 1986; 39:769-73.

8. Woolf PD. Hormonal response to trauma. Crit Care Med 1992;20:21626.

9. Kelly DF, Gonzalo IT, Cohan P, Berman N, Swerdloff R, Wang C. Hypopituitarism following traumatic brain injury and aneurismal subarachnoid haemorrhage: a preliminary report. J Neurosurg 2000:93:743-52.

10. Lieberman SA, Oberoi AL, Gilkison CR, Masel BE, Urban RJ. Prevalence of neuroendocrine dysfunction in patients recovering from traumatic brain injury. J Clin Endocrinol Metab 2001;86:2752-6.

11. Bondanelli M, De Marinis L, Ambrosio MR, Monesi M, Valle D, Zatelli MC. Occurrence of pituitary dysfunction following traumatic brain injury. J Neurotr 2004; 21:685-96.

12. Aimaretti G, Ambrosio MR, Di Somma C, Gasperi M, Cannavo S, Scaroni C. Residual pituitary function after brain injury-induced hypopituitarism: a prospective 12-month study. J Clin Endocrinol Metab 2005;90:6085-92.

13. Agha A, Rogers B, Sherlock M. Anterior pituitary dysfunction in survivors of traumatic brain injury. J Clin Endocrinol Metab 2004;89:4929-36.

14. Klose M, Juul A, Struck J, Morgenthaler G, Kostejanetz M, FeldRasmussen $U$. Acute and long-term pituitary insufficiency in traumatic brain injury: a prospective single centre study. Clin Endocrinol 2007;67:598-606.

15. Krahulík D, Zapletalová J, Fryšák Z, Vaverka M. Dysfunction of hypothalamo-hypophyseal axis after traumatic brain injury in adults. J Neurosurg 2010;113(3):581-4

16. Sockalosky JJ, Kriel RL, Krach LE, Sheehan M. Precocious puberty after traumatic brain injury. J Pediat 1987;110(3):373-7.

17. Goldman M, Shahar E, Sack J. Assessment of endocrine function in children folowing severe head trauma. Pediat Neurol 1997;17(4):339-43.

18. Niederland T, Makovi H, Gál V, Andéka B, Ábrahám CS, Kovács J. Abnormalities of pituitary function after traumatic brain injury in children. J Neurotrauma 2007;24: 119-127.

19. Einaudi S, Matarazzo P, Peretta P, Grossetti P, Giordano F, Altare F. Hypothalamohypophyseal dysfunction after traumatic brain injury in children and adolescents: A preliminary retrospective and prospective study. J Pediat Endocrinol Metab 2006;19:692-703.

20. Poomthavorn P, Maixner W, Zacharin M. Pituitary function in paediatric survivors of severe traumatic brain injury. Arch Dis Child 2008;3:133-7.

21. Aleksijevic D, Zapletalová J, Smolka V, Klásková E, Wiedermann J, Krahulík D, Vaverka M, Fryšák Z. Neuroendokrinní dysfunkce u dětí a dospívajících po úrazu mozku. Cesk Slov Neurol N 2010;106(4):40914.

22. Marshall WA, Tanner JM. Variations in the pattern of pubertal changes in girls. Arch Dis Child 1969;44:291-303.

23. Marshall WA, Tanner JM. Variations in the pattern of pubertal changes in boys. Arch Dis Child 1970;45:13-23.

24. Bláha P, Vígnerová J, Riedlová J, Kobzová J, Krejčovský L. VI. Celostátní antropologický výzkum dětí a mládeže 2001 (National anthropological research of children and adolescents 2001). Čes-slov Pediat 2003;58(12):766-70.

25. Greuliche WW, Pyle SI. Radiographic atlas of skeletal development of the hand and wrist. $2^{\text {nd }}$ edition, Stanford, CA: Stanford University Press;1959.p.156-9.

26. Urban RJ, Harris P, Massel B. Anterior hypopituitarism following traumatic brain injury. Brain Inj 2005;19(5):349-58. 